\title{
HD 60532, a planetary system in a 3:1 mean motion resonance
}

\author{
J. Laskar ${ }^{1}$ and A. C. M. Correia ${ }^{1,2}$ \\ 1 ASD, IMCCE, CNRS-UMR8028, Observatoire de Paris, UPMC, 77 avenue Denfert-Rochereau, 75014 Paris, France \\ e-mail: laskar@imcce.fr \\ 2 Departamento de Física, Universidade de Aveiro, Campus de Santiago, 3810-193 Aveiro, Portugal \\ e-mail: correia@ua.pt
}

Received 20 January 2009 / Accepted 3 February 2009

ABSTRACT

\begin{abstract}
A recent paper has reported that there is a planetary system around the star HD 60532, composed of two giant planets in a possible 3:1 mean motion resonance, which should be confirmed within the next decade. Here we show that the analysis of the global dynamics of the system already allows this resonance to be confirmed. The present best fit to the data corresponds to this resonant configuration, and the system is stable for at least 5 Gyr. The 3:1 resonance is so robust that stability is still possible for a wide variety of orbital parameters around the best-fit solution and would also be if the inclination of the system orbital plane is as small as $15^{\circ}$ with respect to the plane of the sky. Moreover, if the inclination is taken as a free parameter in the adjustment to observations, we find an inclination $\sim 20^{\circ}$, which corresponds to $M_{b}=3.1 M_{\text {Jup }}$ and $M_{c}=7.4 M_{\text {Jup }}$ for the planetary companions.
\end{abstract}

Key words. stars: individual: HD 60532 - stars: planetary systems - techniques: radial velocities - methods: numerical

\section{Introduction}

A recent paper (Desort et al. 2008, hereafter DES08) reported detecting two planetary mass companions around the F type star HD 60532. Using the data acquired with the HARPS spectrograph based on the 3.6-m ESO telescope at La Silla Observatory and a two-Keplerian fit to the data, the orbits of the two bodies were determined, corresponding to minimum masses of $1.03 M_{\text {Jup }}$ and $2.46 M_{\text {Jup }}$ with 201-day and 604-day periods, respectively. The dynamical study done in DES08 suggested a possible 3:1 resonance, but the stability of the results seemed to be questionable, as small variations in the semi-major axis led to a non resonant solution (DES08). Therefore, the authors concluded that the existence of a mean motion resonance would require an additional decade of observations before being confirmed.

The presence of two or more interacting planets in a system dramatically increases our ability to constrain and understand the processes of planetary formation and evolution. The dynamical analysis of these systems is then very useful, first for constraining the system evolution history and second for determining the system "structure" in terms of orbital content. Among the known multi-planet systems, a significant fraction present strong interactions and are trapped in mean motion resonances. The resonances between planets are believed to be formed after the inward or outward migration of the planets during the early stages of the system's formation (e.g. Tsiganis et al. 2005).

By performing a detailed dynamical analysis of the HD 60532 system in conformity with the radial-velocity observations, we can confirm this system in a 3:1 mean-motion resonance and provide some constraints on the inclination of the system orbital plane with respect to the plane of the sky. In Sect. 2 we analyze the observational data obtained with the HARPS spectrograph. The 3:1 resonance and the dynamical analysis of the system are discussed in Sects. 3 and 4. Section 5 contains some additional constraints, and conclusions drawn in Sect. 6.

\section{Orbital solution for the HD 60532 system}

The 147 published radial-velocity data points of HD 60532 (DES08) were taken with the HARPS spectrograph from February 2006 until June 2008. A two-companion Keplerian solution is provided in DES08, but because of the strong mutual interactions between the two planets, these parameters present strong variations and cannot be used in dynamical studies unless the initial longitudes of both planets are given. Using the iterative Levenberg-Marquardt method (Press et al. 1992), we thus re-fitted the complete set of radial velocities with a 3-body Newtonian model, assuming co-planar motion perpendicular to the plane of the sky, similarly to what has been done for the system HD 45364 (Correia et al. 2009). This fit yields an adjustment of $\sqrt{\chi^{2}}=4.41$ and $\mathrm{rms}=4.38 \mathrm{~ms}^{-1}$, slightly better than the twoKeplerian model from DES08. The set of orbital parameters for this system $\left(\mathcal{S}_{1}\right)$ is given in Table 1 .

Still assuming co-planar motion, we then release the perpendicularity constraint by including the inclination as a free parameter. The system readjusts the orbital parameters of the two planets slightly and provides an inclination around $20^{\circ}$ for the best stable fit, although subjected to a large incertitude. By fixing the inclination at this value the new fit yields an adjustment of $\sqrt{\chi^{2}}=4.37$ and $\mathrm{rms}=4.34 \mathrm{~ms}^{-1}$. In this case, the masses are increased by a factor $1 / \sin i=2.92$, i.e., $M_{b}=3.1 M_{\text {Jup }}$ and $M_{c}=7.4 M_{\text {Jup }}\left(\mathcal{S}_{2}\right.$, Table 1$)$. We also attempted to fit the data with a 3-body Newtonian model for which the inclination of the orbital planes, as well as the node of the outer planet orbit, was free to vary. We were able to find a wide variety of configurations, some with very low inclination values for one or both planets, which slightly improved the fit. However, all of these 
Table 1. Orbital parameters for the HD 60532 system $\mathcal{S}_{1}$, obtained with a 3-body Newtonian fit with $i=90^{\circ}$ (top) and for the system system $\mathcal{S}_{2}$, with $i=20^{\circ}$ (bottom) $\left(M_{\star}=1.44 M_{\odot}\right)$.

\begin{tabular}{|c|c|c|c|}
\hline Param. & [unit] & HD 60532 b & HD 60532 c \\
\hline$\sqrt{\chi^{2}}$ & & \multicolumn{2}{|c|}{4.407} \\
\hline $\mathrm{rms}$ & {$[\mathrm{m} / \mathrm{s}]$} & \multicolumn{2}{|c|}{4.380} \\
\hline Date & [JD-2 400 000] & \multicolumn{2}{|c|}{54000.00 (fixed) } \\
\hline V & {$\left[\mathrm{km} \mathrm{s}^{-1}\right]$} & \multicolumn{2}{|c|}{$-0.0049 \pm 0.0004$} \\
\hline$P$ & [day] & $201.46 \pm 0.13$ & $605.28 \pm 2.12$ \\
\hline$\lambda$ & [deg] & $14.48 \pm 0.69$ & $316.23 \pm 0.95$ \\
\hline$e$ & & $0.279 \pm 0.006$ & $0.027 \pm 0.007$ \\
\hline$\omega$ & [deg] & $352.15 \pm 1.08$ & $136.81 \pm 16.34$ \\
\hline$K$ & {$[\mathrm{~m} / \mathrm{s}]$} & $29.63 \pm 0.32$ & $46.80 \pm 0.40$ \\
\hline$i$ & [deg] & 90 (fixed) & 90 (fixed) \\
\hline$M$ & {$\left[M_{\text {Jup }}\right]$} & 1.0484 & 2.4866 \\
\hline$a$ & {$[\mathrm{AU}]$} & 0.7597 & 1.5822 \\
\hline Param. & [unit] & HD 60532 b & HD 60532 c \\
\hline$\sqrt{\chi^{2}}$ & & \multicolumn{2}{|c|}{4.369} \\
\hline $\mathrm{rms}$ & {$[\mathrm{m} / \mathrm{s}]$} & \multicolumn{2}{|c|}{4.342} \\
\hline Date & [JD-2 400000$]$ & \multicolumn{2}{|c|}{54000.00 (fixed) } \\
\hline$V$ & {$\left[\mathrm{~km} \mathrm{~s}^{-1}\right]$} & \multicolumn{2}{|c|}{$-0.0055 \pm 0.0003$} \\
\hline$P$ & [day] & $201.83 \pm 0.14$ & $607.06 \pm 2.07$ \\
\hline$\lambda$ & [deg] & $14.78 \pm 0.66$ & $317.02 \pm 0.93$ \\
\hline$e$ & & $0.278 \pm 0.006$ & $0.038 \pm 0.008$ \\
\hline$\omega$ & [deg] & $352.83 \pm 1.05$ & $119.49 \pm 9.14$ \\
\hline$K$ & {$[\mathrm{~m} / \mathrm{s}]$} & $30.34 \pm 0.32$ & $47.84 \pm 0.44$ \\
\hline$i$ & [deg] & 20 (fixed) & 20 (fixed) \\
\hline$M$ & {$\left[M_{\mathrm{Jup}}\right]$} & 3.1548 & 7.4634 \\
\hline$a$ & [AU] & 0.7606 & 1.5854 \\
\hline
\end{tabular}

Table 2. Fundamental frequencies for the orbital solution $\mathcal{S}_{1}$ (Table 1).

\begin{tabular}{ccrr}
\hline \hline & $\begin{array}{c}\text { Frequency } \\
\% / y r\end{array}$ & $\begin{array}{r}\text { Period } \\
\text { yr }\end{array}$ \\
\hline mean & $n_{b}$ & 653.645983 & 0.550757 \\
motions & $n_{c}$ & 217.563988 & 1.654686 \\
\hline secular & $g_{1}$ & -0.477010 & -754.701 \\
frequencies & $g_{2}$ & -0.009651 & -37303.55 \\
\hline libration frequency & $l_{\theta}$ & 9.015796 & 39.9299 \\
\hline
\end{tabular}

determinations are uncertain, and since we also increased the number of free parameters by three, we cannot say that these solutions present much of an improvement with respect to the sets $\mathcal{S}_{1}, \mathcal{S}_{2}$, presented in Table 1 .

\section{The 3:1 mean motion resonance}

As in DES08, our nominal solution $\mathcal{S}_{1}$ (Table 1) seems to be in a 3:1 resonance. We numerically integrated the orbits of the planets over $100 \mathrm{kyr}$ with the symplectic integrator SABAC4 of Laskar \& Robutel (2001), using a step size of 0.02 years. The frequency analysis of this orbital solution allows then to conclude that it is indeed in a 3:1 mean motion resonance, with resonant argument: $\theta=\lambda_{b}-3 \lambda_{c}+2 \omega_{b}$.

The fundamental frequencies of the systems are the two mean motions $n_{b}$ and $n_{c}$, the two secular frequencies of the periastrons $g_{1}$ and $g_{2}$, and the libration frequency of the resonant argument $l_{\theta}$ (Table 3$)$. Up to the precision of the frequency determinations $\left(\approx 10^{-9}\right)$, we have the resonant relation $n_{b}-3 n_{c}+2 g_{1}=0$.

For the nominal solution $\mathcal{S}_{1}$, the resonant argument $\theta$ is in libration around $180^{\circ}$, with a libration period $2 \pi / l_{\theta}=39.93 \mathrm{yr}$ and an associated amplitude of about 38.138 degrees, with
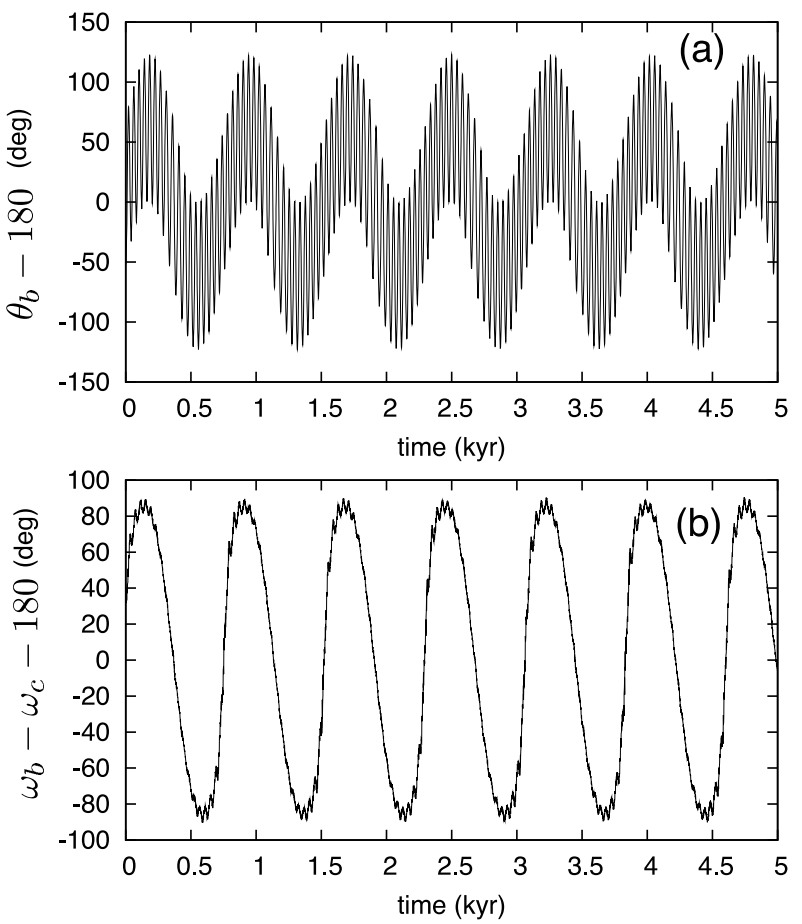

Fig. 1. Variation in the resonant argument, $\theta=\lambda_{b}-3 \lambda_{c}+2 \omega_{b}$ (a) and in the secular argument, $\Delta \omega=\omega_{b}-\omega_{c}(\mathbf{b})$, with time.

Table 3. Quasi-periodic decomposition of the resonant angle $\theta=\lambda_{b}-$ $3 \lambda_{c}+2 \omega_{b}$ for the orbital solution $\mathcal{S}_{1}$ (Table 1 ).

\begin{tabular}{|c|c|c|c|c|c|c|c|}
\hline \multicolumn{5}{|c|}{ Combination } & \multirow{2}{*}{$\begin{array}{c}v_{i} \\
(\mathrm{deg} / \mathrm{yr})\end{array}$} & \multirow{2}{*}{$\begin{array}{c}A_{i} \\
\text { (deg) }\end{array}$} & \multirow{2}{*}{$\begin{array}{c}\phi_{i} \\
\text { (deg) }\end{array}$} \\
\hline$n_{b}$ & $n_{c}$ & $g_{1}$ & $g_{2}$ & $l_{\theta}$ & & & \\
\hline 0 & 0 & -1 & 1 & 0 & 0.4674 & 63.652 & -81.114 \\
\hline 0 & 0 & 0 & 0 & 1 & 9.0158 & 38.138 & 168.018 \\
\hline 0 & 0 & 1 & -1 & 1 & 8.5484 & 35.869 & 159.131 \\
\hline 0 & 0 & 2 & -2 & 1 & 8.0811 & 25.845 & -29.755 \\
\hline 0 & 0 & 3 & -3 & 1 & 7.6137 & 16.868 & 141.358 \\
\hline 0 & 0 & 4 & -4 & 1 & 7.1464 & 10.622 & -47.528 \\
\hline 0 & 0 & 5 & -5 & 1 & 6.6790 & 6.325 & 123.585 \\
\hline 0 & 0 & 6 & -6 & 1 & 6.2116 & 3.509 & -65.301 \\
\hline 0 & 0 & -2 & 2 & 0 & 0.9347 & 2.697 & 107.773 \\
\hline 0 & 0 & -1 & 1 & 1 & 9.4832 & 2.366 & 176.904 \\
\hline 0 & 0 & 7 & -7 & 1 & 5.7443 & 1.872 & 105.812 \\
\hline 0 & 0 & 3 & -3 & 2 & 16.6295 & 1.156 & 39.376 \\
\hline 0 & 0 & 2 & -2 & 2 & 17.0969 & 0.874 & -131.738 \\
\hline 0 & 0 & -2 & 2 & 1 & 9.9505 & 0.874 & 5.791 \\
\hline 0 & 0 & 0 & 0 & 2 & 18.0316 & 0.828 & 66.035 \\
\hline 0 & 0 & 8 & -8 & 1 & 5.2769 & 0.964 & -83.074 \\
\hline 0 & 0 & 4 & -4 & 2 & 16.1622 & 0.805 & -149.510 \\
\hline 0 & 0 & 1 & -1 & 2 & 17.5642 & 0.499 & -122.851 \\
\hline 0 & 0 & -3 & 3 & 1 & 10.4179 & 0.517 & -165.323 \\
\hline 0 & 0 & -3 & 3 & 0 & 1.4021 & 0.433 & -63.341 \\
\hline 0 & 0 & 9 & -9 & 1 & 4.8096 & 0.482 & 88.040 \\
\hline 1 & -1 & 0 & 0 & 0 & 436.0820 & 0.395 & -173.181 \\
\hline
\end{tabular}

We have $\theta(t)=180+\sum_{i=1}^{N} A_{i} \cos \left(v_{i} t+\phi_{i}\right)$. All terms are identified as integer combinations of the fundamental frequencies given in Table 2 , which is the signature of a regular motion.

multiples additional terms of significant amplitude of the form $k(g 1-g 2)+l_{\theta}$, with $k$ integer (Fig. 1a, Table 3$)$. For the complete solution, the libration amplitude can reach 123.16 degrees because additional periodic terms are present. In Table 3, we provide a quasi-periodic decomposition of the resonant angle $\theta$ in terms of decreasing amplitude. All the quasi-periodic terms are 

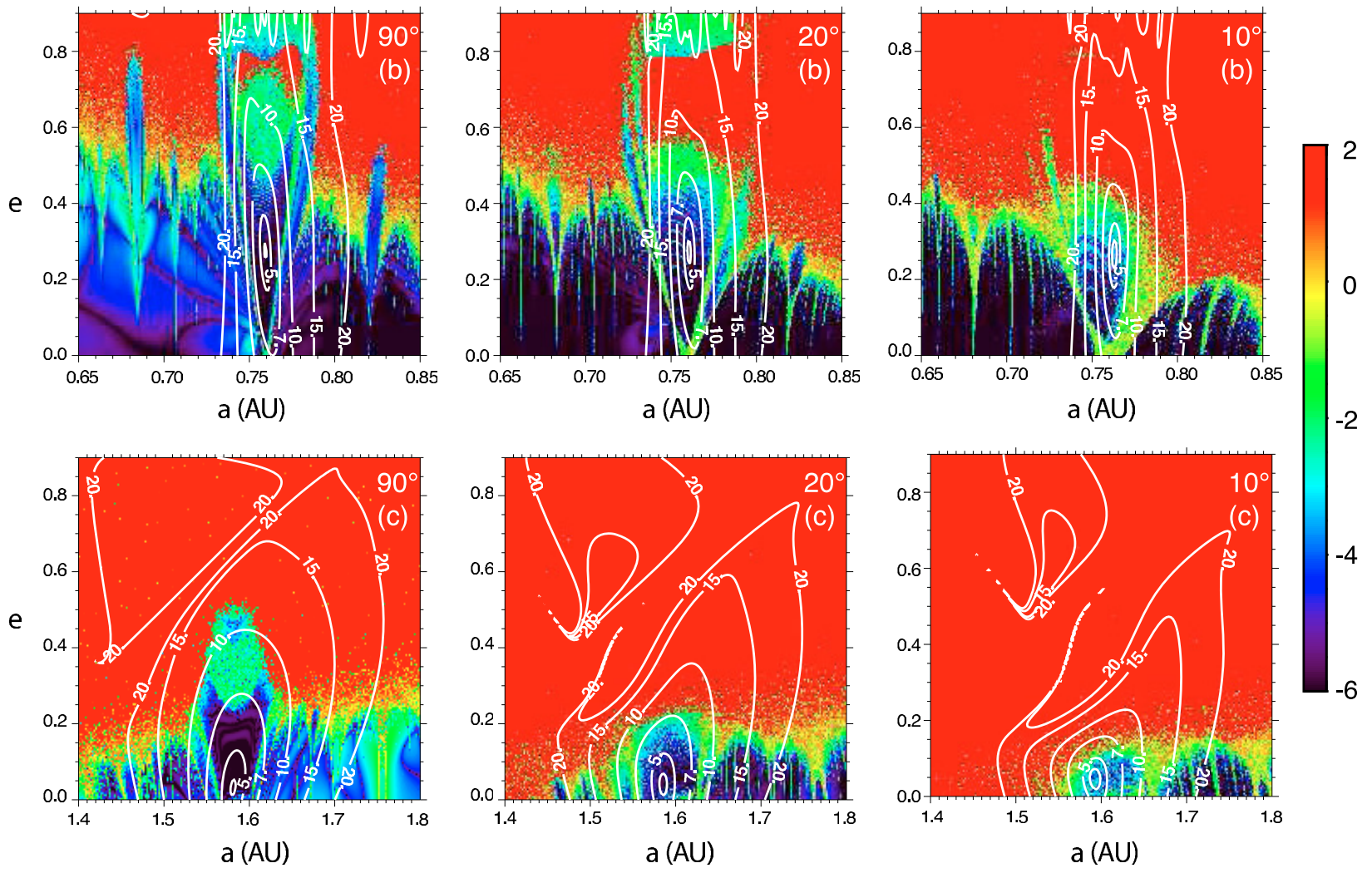

Fig. 2. Stability analysis of the HD 60532 planetary system for different values of the inclination $\left(90^{\circ}, 20^{\circ}, 10^{\circ}\right)$. For a fixed orbital inclination and initial condition of the outer and inner planet, the phase space of the system is explored by varying the semi-major axis $a_{k}$ and eccentricity $e_{k}$ of the other planet, b) and c) respectively. The step size is $0.002 \mathrm{AU}$ in semi-major axis and 0.005 in eccentricity. For each initial condition, the full system is integrated numerically over $10 \mathrm{kyr}$ and, as in (Correia et al. 2005, 2009), a stability criterion is derived with the frequency analysis of the mean longitude (Laskar 1990, 1993). The "red" zone corresponds to highly unstable orbits, while the "dark blue" region can be assumed to be stable on a billion-year timescale. The contour curves indicate the value of rms obtained for each choice of parameters. It is remarkable that in the present fit, there is perfect correspondence between the zone of minimal rms and the 3:1 stable resonant zone, in "dark blue".

easily identified as integer combinations of the fundamental frequencies (Table 3). Since the resonant angle is modulated by a relatively short period of about 38 years and high harmonics of shorter periods, the observation of the system over a few additional years may provide an estimate of the libration amplitude and thus a strong constraint on the parameters of the system.

As in (Correia et al. 2009), both periastrons precess with mean frequency $g_{1}$ that is retrograde, with a period of 754.701 years. The two periastrons are thus locked in an antipodal state, and the difference $\Delta \omega=\omega_{b}-\omega_{c}$ is in libration around $180^{\circ}$ with an amplitude of $90^{\circ} .7$ (Fig. 1b). As a result, the arguments $\theta^{\prime}=\lambda_{b}-3 \lambda_{c}+2 \omega_{c}$ and $\theta^{\prime \prime}=\lambda_{b}-3 \lambda_{c}+\omega_{b}+\omega_{c}$ librate around $180^{\circ}$ and $0^{\circ}$, respectively, with the same libration frequency $l_{\theta}$.

\section{Stability analysis}

To analyze the stability of the nominal solution and confirm the presence of the 3:1 resonance, we performed a global frequency analysis (Laskar 1993) in the vicinity of the nominal solution (Fig. 2), in the same way as achieved for the HD 202206 (Correia et al. 2005) and HD 45364 (Correia et al. 2009) systems. For each planet, the system is integrated on a regular $2 \mathrm{D}$ mesh of initial conditions, with varying semi-major axis and eccentricity, while the other parameters are retained at their nominal values. The solution is integrated over $10 \mathrm{kyr}$ for each initial condition and a stability indicator is computed as the variation in the measured mean motion over two consecutive $5 \mathrm{kyr}$ intervals of time.
The results, for different values of the inclination of the system $\left(i=90^{\circ}, 20^{\circ}, 10^{\circ}\right)$, are reported in color in Fig. 2, where "red" represents the strongly chaotic trajectories, and "dark blue" the extremely stable ones. In all these plots, the 3:1 resonant island is clearly highlighted and appears to be very stable until the inclination reaches $i=10^{\circ}$, for which most of the resonant island is destabilized. Indeed, a numerical integration of the fitted solution for 10 degrees inclination led to a disruption of the system in $584 \mathrm{Myr}$.

It is remarkable that, as for HD 45364 (Correia et al. 2009), there is perfect coincidence between the stable $3: 1$ resonant islands, and curves of minimal rms obtained by comparison with the observations. Moreover, for our preferred solutions, with inclination ranging from 90 to 15 degrees, the resonant island extends to high values of the rms of about $7 \mathrm{~m} / \mathrm{s}$, which reinforces the confidence that the present system is in a 3:1 resonant state.

The present dynamical analysis also indicates that this 3:1 mean motion resonance is stable over Gyr timescales. We have also directly tested this stability by performing a numerical integration of the systems $\mathcal{S}_{1}$ and $\mathcal{S}_{2}$ (Table 1) over 5 Gyr using the symplectic integrator SABAC4 and a step size of 0.02 years. Both solutions, $\mathcal{S}_{1}, \mathcal{S}_{2}$, remain stable over $5 \mathrm{Gyr}$, although their orbital elements present large variations because of the strong gravitational interactions between the two planets. For the nominal solution $\mathcal{S}_{1}$, the eccentricity of the inner planet oscillates within $0.115<e_{b}<0.301$, while we have $0.0165<e_{c}<0.143$ for the outer planet. The secular variations in the orbital parameters are mostly driven by the rapid secular frequency $g_{1}$, of 


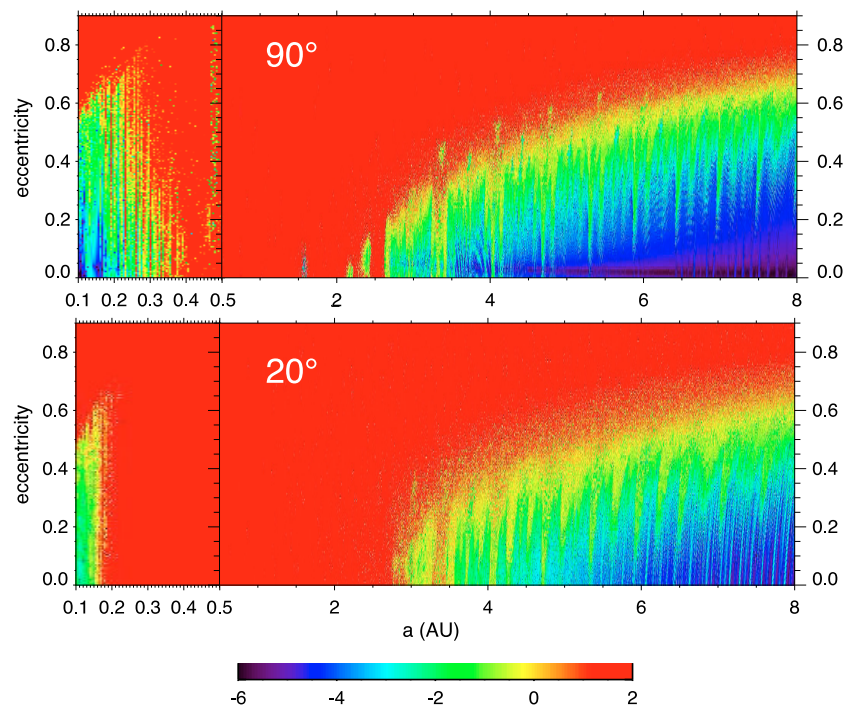

Fig. 3. Possible location of an additional planet in the HD 60532 system. For 90 (top) and 20 degrees (bottom) inclination, the stability of a small mass particle in the HD 60532 system is analyzed, for various semimajor axis and eccentricity, and for $K=10^{-6} \mathrm{~m} / \mathrm{s}$. The stable zones where additional planets could be found are the dark blue regions.

period $2 \pi / g_{1} \approx 754 \mathrm{yr}$ (Table 3 ). These secular variations in the orbital elements occur much more rapidly than in our Solar System, which may enable them to be detected directly from observations.

\section{Additional constraints}

The stability analysis summarized in Fig. 2 shows, as in HD 45364 (Correia et al. 2009), very good agreement between the $3: 1$ resonant islands and the rms contour curves. We can thus assume that the dynamics of the two known planets is not disturbed much by the presence of some large planet close by.

We tested the possibility of an additional planet in the systems $\mathcal{S}_{1}$ and $\mathcal{S}_{2}$ by including a third planet, with varying semimajor axis and eccentricity over a wide range, and by performing stability analysis (Fig. 3). The test was done for a fixed $K$ value: first with an asteroid size object $\left(K=10^{-6} \mathrm{~m} / \mathrm{s}\right)$ (Fig. 3), and then for an Earth-size object $(K=0.1 \mathrm{~m} / \mathrm{s})$ without noticeable change in the dynamics.

From this analysis, one can see (Fig. 3) that, for $\mathcal{S}_{1}\left(i=90^{\circ}\right)$, the zone of instability extends from about $0.2 \mathrm{AU}$ to about $4 \mathrm{AU}$, while for $\mathcal{S}_{2}\left(i=20^{\circ}\right)$, no planet can be stable from $0.1 \mathrm{AU}$ to about $6 \mathrm{AU}$. The eventual discovery of an additional planet in this range would thus constrain the inclination of the system with respect to the plane of view, and hence the planetary masses. At $i=90^{\circ}$, the libration period of the $3: 1$ resonant argument is $39.93 \mathrm{yr}$, but this period depends strongly on the planetary masses, and thus on the inclination $i$ of the system (Table 4). We computed the libration period $P_{\theta}$ for the best fit obtained at various inclinations, $i=90,30,20,15,10$ degrees. At $i=20^{\circ}$, we have $P_{\theta}=23.17 \mathrm{yr}$. As the amplitude of the libration is large, we can expect that, over the next decade, this resonant libration period will be constrained, allowing good determination of the inclination and planetary masses. Indeed, if we extrapolate the differences of radial velocities for various inclination hypotheses ( $i=30,20,15,10$ degrees), compared with the nominal solution $\mathcal{S}_{1}$, it clearly appears that the inclination of the system will be determined within a few years with the HARPS data (Fig. 4).
Table 4. Change in the mass factor $(1 / \sin i)$ and libration period $P_{\theta}$ with inclination $(i)$.

\begin{tabular}{crrrrr}
\hline \hline$i$ & $90^{\circ}$ & $30^{\circ}$ & $20^{\circ}$ & $15^{\circ}$ & $10^{\circ}$ \\
\hline $1 / \sin i$ & 1.00 & 2.00 & 2.92 & 3.86 & 5.76 \\
$P_{\theta}(\mathrm{yr})$ & 39.93 & 28.88 & 23.17 & 19.57 & 15.35 \\
\hline
\end{tabular}

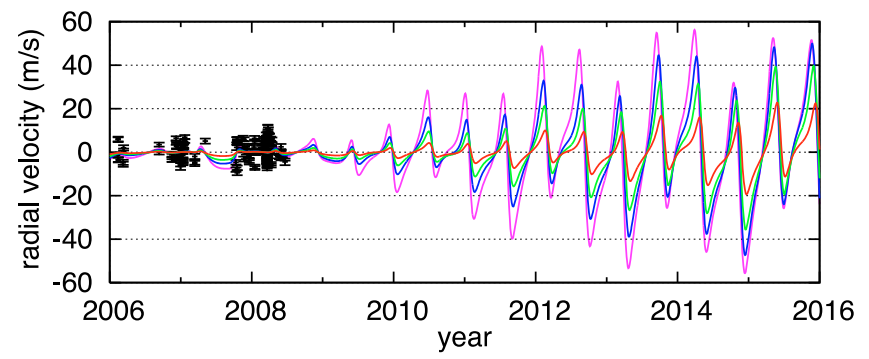

Fig. 4. Radial velocity signature of the inclination $(i)$. The difference with respect to the nominal solution (at $i=90^{\circ}$ ) are plotted over time for $i=30^{\circ}$ (red), $20^{\circ}$ (green), $15^{\circ}$ (blue), and $10^{\circ}$ (violet). The available HARPS residual data are also plotted with their error bars.

\section{Discussion and conclusion}

We have re-analyzed the dynamics of the two planets system HD 60532, as first reported in DES08. In contrast to the conclusions of this previous work, we believe that the global dynamical study presented here (Fig. 2) allows the unambiguous conclusion that the system is in a 3:1 mean motion resonance.

Moreover, thanks to the strong dynamical interaction present in this system, we could also fit the inclination, which led to a most probable value of 20 degrees, while our dynamical analysis set up a stability constraint to $15^{\circ} \leq i \leq 90^{\circ}$. The confidence in the existence of the $3: 1$ resonance is enhanced by the fact that the system remains in resonance for all the fits that we performed, from 90 to 15 degrees inclination. In the nominal solution $\left(\mathcal{S}_{1}\right.$, Table 1), the resonant angle $\theta=\lambda_{b}-3 \lambda_{c}+2 \omega_{b}$ is in libration around $180^{\circ}$, with a libration period of 39.93 years and an associated amplitude of $38^{\circ} .14$, although the main oscillation of this argument is driven by the secular term $g_{1}-g_{2}$ with an associated amplitude of $63^{\circ} .65$, the total amplitude of the libration being $123^{\circ} .16$, due to additional harmonics.

The planet-planet interactions in this system are important, because of this 3:1 resonance. The dependence of the libration period with the mass factor (Table 4) should allow determination of the inclination of the orbital planes and thus of the masses of both planets within the next decade (Fig. 4).

Acknowledgements. We acknowledge support from the Fundação Calouste Gulbenkian (Portugal) and French PNP-CNRS. This work was started during a stay at Geneva Observatory, where discussions with M. Mayor and S. Udry were very helpful.

\section{References}

Correia, A. C. M., Udry, S., Mayor, M., et al. 2005, A\&A, 440, 751

Correia, A. C. M., Udry, S., Mayor, M., et al. 2009, A\&A, 496, 521

Desort, M., Lagrange, A.-M., Galland, F., et al. 2008, A\&A, 491, 883

Laskar, J. 1990, Icarus, 88, 266

Laskar, J. 1993, Physica D, 67, 257

Laskar, J., \& Robutel, P. 2001, Celest. Mech. Dyn. Astron., 80, 39

Press, W. H., Teukolsky, S. A., Vetterling, W. T., \& Flannery, B. P. 1992, Numerical recipes in FORTRAN, The art of scientific computing (Cambridge: University Press), 2nd edn.

Tsiganis, K., Gomes, R., Morbidelli, A., \& Levison, H. F. 2005, Nature, 435, 459 